\title{
Risk factors for superficial incisional surgical site infection after gastrectomy: analysis of patients enrolled in a prospective randomized trial comparing skin closure methods
}

\author{
Shunji Endo ${ }^{1}$ Toshimasa Tsujinaka ${ }^{2,3}$ Kazumasa Fujitani ${ }^{2,4} \cdot$ Junya Fujita $^{5,6}$ • \\ Shigeyuki Tamura ${ }^{7}$. Makoto Yamasaki ${ }^{8} \cdot$ Shogo Kobayashi $^{8,9} \cdot$ Yusuke Akamaru $^{10}$. \\ Tsunekazu Mizushima ${ }^{8} \cdot$ Junzo Shimizu $^{11} \cdot$ Koji Umeshita $^{8} \cdot$ Toshinori Ito $^{8} \cdot$ \\ Masaki Mori $^{8} \cdot$ Yuichiro Doki $^{8}$
}

Received: 12 December 2014/ Accepted: 26 March 2015/Published online: 11 April 2015

(C) The International Gastric Cancer Association and The Japanese Gastric Cancer Association 2015

\begin{abstract}
Background Surgical site infection is one of the commonest complications of gastrointestinal surgery. The nature of surgical procedures and wound closure methods may influence the incidence of superficial incisional surgical site infection. Patients enrolled in a prospective randomized controlled trial comparing skin closure methods are the best subjects for analyzing surgical site infection risk.
\end{abstract}

On behalf of the Clinical Study Group of Osaka University on Section of Risk Management.

Shunji Endo

endo-s@higashiosaka-hosp.jp

1 Department of Gastroenterological Surgery, Higashiosaka City General Hospital, Osaka, Japan

2 Department of Surgery, National Hospital Organization Osaka National Hospital, Osaka, Japan

3 Department of Surgery, Kaizuka City Hospital, Osaka, Japan

4 Department of Surgery, Osaka General Medical Center, Osaka, Japan

5 Department of Gastroenterological Surgery, Toyonaka Municipal Hospital, Osaka, Japan

6 Department of Surgery, NTT WEST Osaka Hospital, Osaka, Japan

7 Department of Surgery, Kansai Rosai Hospital, Hyogo, Japan

8 Department of Gastroenterological Surgery, Osaka University Graduate School of Medicine, Osaka, Japan

9 Department of Surgery, Osaka Medical Center for Cancer and Cardiovascular Diseases, Osaka, Japan

10 Department of Surgery, Japan Community Health Care Organization Osaka Hospital, Osaka, Japan

11 Department of Surgery, Osaka Rosai Hospital, Osaka, Japan
Method From a cohort of 1080 patients who had been enrolled in our previous randomized controlled trial, data for 685 patients who had undergone elective open total gastrectomy or distal gastrectomy for gastric cancer were extracted. The incidences of superficial incisional surgical site infection after total gastrectomy and distal gastrectomy were compared and risk factors for superficial incisional surgical site infection were investigated by univariate analyses using logistic regression models.

Results In all, 42 patients (6.1\%) developed superficial incisional surgical site infections after gastrectomy; 15 of 288 patients $(5.2 \%)$ developed these infections after total gastrectomy, and 27 of 397 patients (6.8\%) developed these infections after distal gastrectomy-these differences are not significant. According to univariate analysis, age (75 years or older or younger than 75 years) was the only risk factor for superficial incisional surgical site infections $(P=0.049)$. There was a tendency for the incidence of superficial incisional surgical site infection to increase in parallel with age.

Conclusion The incidence of superficial incisional surgical site infection did not differ between total gastrectomy and distal gastrectomy. Advanced age was the only identified risk factor for superficial incisional surgical site infections after gastrectomy.

Keywords Stomach neoplasms · Gastrectomy - Surgical wound infection

\section{Introduction}

A surgical site infection (SSI) is a postoperative infection that occurs in the part of the body where a surgical procedure has been performed. According to the surgical 
patient component of the 1999 Centers for Disease Control and Prevention (CDC) National Nosocomial Infection Surveillance System manual, the term "surgical site infection" (SSI) includes superficial incisional, deep incisional, and organ/space SSIs [1]; however, the focus has mainly been on superficial incisional SSIs.

Various incidences of superficial incisional SSI following gastrectomy has been reported; they differ greatly because of differences in background factors, operative procedures, and assessment processes. According to the National Healthcare Safety Network of the CDC in the USA, SSIs at primary incision sites occurred following $2.2 \%$ of all gastric surgery procedures in 2006-2008 [2], whereas the Japanese Healthcare Associated Infections Surveillance [3] reported a figure of $3.5 \%$ in 2012 in Japan. The Osaka Gastrointestinal Cancer Chemotherapy Study Group has performed randomized controlled trials (RCTs) in which the primary end points were incidences of SSIs: it found that superficial incisional SSIs occurred in 15 of 231 patients $(6.5 \%)$ following total gastrectomy [4] and in six of 355 patients $(1.7 \%)$ following distal gastrectomy [5]. Although skin and fascia closing methods were not controlled, these data suggest that total gastrectomy causes more superficial incisional SSIs than does distal gastrectomy. There are no published prospective studies in which many risk factors for SSI were well controlled that compare the incidence of superficial incisional SSIs after total gastrectomy and distal gastrectomy.

Recently, we performed a multicenter open-label phase III RCT to compare subcuticular sutures with staples for skin closure after open gastrointestinal surgery: our results concerning wound complications have been reported (UMIN00002480) [6]. Because only patients who underwent elective open surgery were included, patients with high risk of SSI were excluded, and fascia and skin closure methods were controlled, the patients enrolled in this trial seemed ideal candidates for evaluating risk factors for superficial incisional SSI and for comparing surgical procedures.

We extracted data from the relevant cohort of our previous trial to compare the incidence of superficial incisional SSIs after total gastrectomy and distal gastrectomy and to investigate risk factors for superficial incisional SSI.

\section{Patients and methods}

\section{Patients}

From 1 June 2009 to 28 February 2012, we conducted a large-scale, multicenter open-label phase III RCT in which 1080 patients from 24 institutions in Japan were enrolled. Eligibility criteria for this trial were patients undergoing elective upper or lower gastrointestinal tract surgery aged 20 years or older, and with adequate organ function. Patients requiring emergency or laparoscopic surgery; with a history of laparotomy via a midline incision; taking corticosteroid drugs long term; with active infections such as peritonitis, pneumonia, or urinary tract infection; with massive ascites; with coagulopathy or other disorders that would preclude study participation; with uncontrolled or insulin-treated diabetes; with mental illness; with poor general condition; or with severe cardiopulmonary disease; or who were otherwise deemed by surgeons to be inappropriate for participation were excluded. The institutional review boards of all participating hospitals approved the protocol. All patients provided written informed consent before randomization. From the cohort of the enrolled patients, data for 685 patients who had undergone elective open total gastrectomy or distal gastrectomy for gastric cancer were extracted for this analysis (Fig. 1). Among them, 288 patients had undergone total gastrectomy and 397 had undergone distal gastrectomy.

In the original trial, patients were randomly assigned to receive either subcuticular sutures or staples for skin closure and were balanced according to institution, sex, and type of surgery (i.e., upper or lower gastrointestinal tract open surgery). All participating institutions were asked to follow the guidelines concerning prevention of SSIs issued by the CDC [1]. Surgical gloves and instruments were changed before wound closure. Absorbable sutures were used to approximate the fascia, and the subcutaneous space was irrigated with saline without added antibiotics. Approximation of the fat layer was not allowed. Drain placement through a separate incision away from the operative incision was permitted; however, drainage of the wound was not allowed. The skin preparation techniques, prophylactic antibiotic administration, the volume of saline used for intra-abdominal irrigation, the dressing methods, the timing of postoperative staple removal, perioperative care, and wound management were according to the standards of each participating institution.

\section{Definition of SSI}

The responsible surgeon checked for the presence or absence of SSIs everyday during the hospital stay and at every outpatient visit until 30 days after surgery. Superficial incisional SSI was defined according to the standardized criteria of the National Nosocomial Infection Surveillance System by the CDC [1] as an infection involving only skin or subcutaneous tissue of an incision with at least one of the following: (1) purulent drainage, with or without laboratory confirmation, from the superficial incision; (2) organisms isolated from an aseptically obtained culture of fluid or tissue from the superficial 
Fig. 1 CONSORT diagram showing eligibility criteria. GI gastrointestinal, SSI surgical site infection

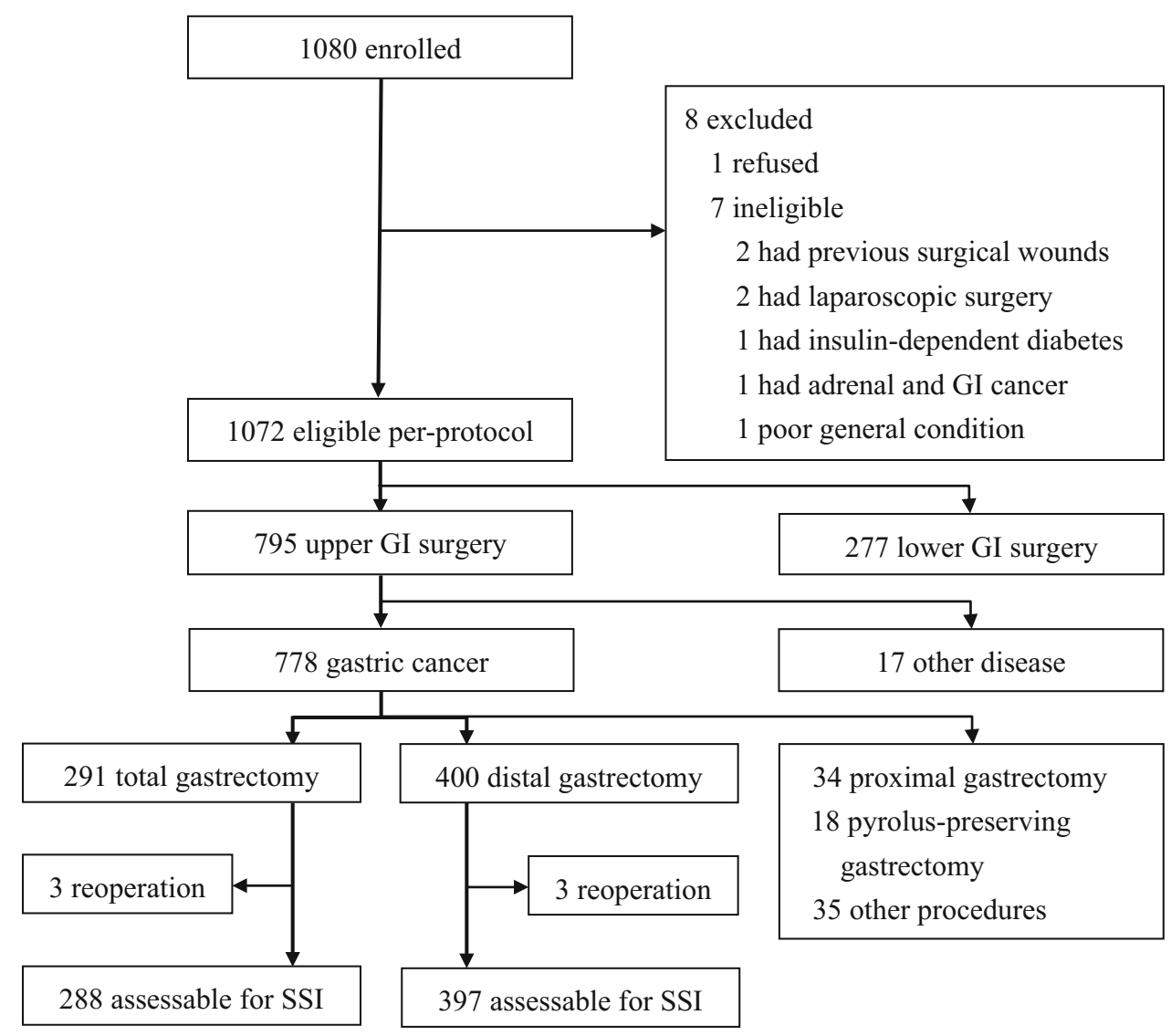

incision; (3) at least one of pain or tenderness, localized swelling, redness, or heat as signs or symptoms of infection and the superficial incision is deliberately opened by the surgeon, unless the incision is culture negative; or (4) diagnosis of superficial incisional SSI by the surgeon or attending physician.

In the original trial, the presence or absence of deep incisional and organ/space SSIs was monitored; however, the evaluation criteria for these SSIs were not clearly defined, and an index of severity, such as Dindo-Clavien scores, was not used. Because there were major differences between institutions regarding the incidences of these SSIs, only the incidence of superficial incisional SSI was evaluated in this study.

\section{Variables for risk analysis}

The following clinical and operative variables were investigated as possible risk factors for SSIs: sex, age (75 years or older or younger than 75 years), hemoglobin concentration $(10 \mathrm{~g} / \mathrm{dL}$ or higher or below $10 \mathrm{~g} / \mathrm{dL})$, C-reactive protein concentration $(0.1 \mathrm{mg} / \mathrm{dL}$ or higher or below $0.1 \mathrm{mg} / \mathrm{dL})$, prognostic nutritional index $[10 \times$ serum albumin concentration $(\mathrm{mg} / \mathrm{dL})+0.005 \times$ lymphocyte count $\left(/ \mathrm{mm}^{3}\right), 40$ or higher or below 40] [7], body mass index (BMI) [body weight (kg)/height $(\mathrm{m})^{2}, 25$ or higher or below 25], thickness of subcutaneous fat (thick or normal/thin according to the surgeon's subjective assessment), extent of gastric resection (total or distal gastrectomy), other organ resection (yes or no), skin closure (subcuticular suture or staples), operation time (240 min or longer or less than $240 \mathrm{~min}$ ), estimated blood loss (300 g or more or less than $300 \mathrm{~g}$ ), duration of antibiotic prophylaxis ( 2 days or more or 1 day), presence of a drain (yes or no), anticoagulation therapy (yes or no), and institution (high-volume institutions that had recruited more than 50 patients or low-volume institutions with fewer than 50 patients).

\section{Statistical analysis}

Quantitative variables were compared between total gastrectomy and distal gastrectomy using the Mann-Whitney $U$ test. Qualitative variables and the incidences of superficial incisional SSI were compared using the $\chi^{2}$ test or Fisher's exact probability test. Risk factors for superficial incisional SSI were subjected to univariate analyses using logistic regression models to assess the effects of the factors on SSI. $P$ values of less than 0.050 were considered statistically significant. Analyses were performed using 
JMP (version 11.0.0 for Windows; SAS Institute, Cary, NC, USA).

\section{Results}

The baseline clinical and operative characteristics of the 685 patients who had undergone elective open total gastrectomy or distal gastrectomy for gastric cancer and who were eligible for assessment of superficial incisional SSIs are shown in Table 1. Patients who had undergone total gastrectomy were more likely to have lower BMI, other organ resection, longer operation time, greater intraoperative blood loss, and placement of drains than those who had undergone distal gastrectomy. Superficial incisional SSIs occurred in 42 patients $(6.1 \%)$, including 15 patients $(5.2 \%)$ following total gastrectomy and 27 patients (6.8 \%) following distal gastrectomy. The incidences of superficial incisional SSIs did not differ between the two procedures $(P=0.387)$.

According to univariate analysis of possible risk factors for superficial incisional SSIs, patients aged 75 years or older had significantly higher incidences of superficial incisional SSIs than did younger patients $(P=0.049)$ (Table 2). No other factors were identified as significant risk factors.
The relationship between age and the incidence of superficial incisional SSIs was further investigated by dividing age into four categories: namely, the young (64 years or younger), the young old (65-74 years), the old old (75-84 years), and the oldest old (85 years or older) [8]. The incidence of superficial incisional SSIs tended to increase in parallel with age; however, the differences between these age groups were not significant (Table 3).

Additionally, the incidence of superficial incisional SSIs was analyzed for four BMI categories: namely, underweight $\left(\mathrm{BMI}<18.5 \mathrm{~kg} / \mathrm{m}^{2}, \quad n=80\right)$, normal range $\left(18.5 \mathrm{~kg} / \mathrm{m}^{2} \leq \mathrm{BMI}<25 \mathrm{~kg} / \mathrm{m}^{2}, \quad n=445\right)$, overweight $\left(25 \mathrm{~kg} / \mathrm{m}^{2} \leq \mathrm{BMI}<30 \mathrm{~kg} / \mathrm{m}^{2}, \quad n=105\right), \quad$ and obesity $\left(\mathrm{BMI} \geq 30 \mathrm{~kg} / \mathrm{m}^{2}, n=15\right)$; BMI data were unavailable for 40 patients. The incidences for these BMI categories were $5.0 \%$ (4/80), $6.5 \%(29 / 445), 5.7 \%(6 / 105)$ and $6.7 \%(1 / 15)$, respectively; the differences between these groups were not significant $(P=0.953)$.

\section{Discussion}

SSI, one of the commonest complications following gastrointestinal surgery, may lead to prolonged hospital stay, increased health care costs, a greater workload for staff, and a deteriorated quality of life. Surgeons should prioritize

Table 1 Clinical and operative characteristics of study patients

\begin{tabular}{|c|c|c|c|}
\hline Variable & Total gastrectomy $(n=288)$ & Distal gastrectomy $(n=397)$ & $P$ \\
\hline Sex: male/female & $212 / 76$ & $272 / 125$ & 0.148 \\
\hline Age (years) & $67(60-74)^{\mathrm{c}}$ & $68(61-74)^{\mathrm{c}}$ & 0.109 \\
\hline Hemoglobin $(\mathrm{g} / \mathrm{dl})$ & $12.9(11.0-14.1)^{\mathrm{c}}$ & $13.1(11.3-14.3)^{\mathrm{c}}$ & 0.373 \\
\hline $\mathrm{CRP}^{\mathrm{a}}(\mathrm{mg} / \mathrm{dl})$ & $0.1(0.0-0.2)^{\mathrm{c}}$ & $0.1(0.0-0.2)^{\mathrm{c}}$ & 0.184 \\
\hline PNI & $49.0(45.1-52.5)^{\mathrm{c}}$ & $49.5(45.5-53.2)^{\mathrm{c}}$ & 0.301 \\
\hline ASA classification: $1 / 2 / 3$ & $117 / 162 / 9$ & $156 / 222 / 19$ & 0.560 \\
\hline $\mathrm{BMI}^{\mathrm{b}}\left(\mathrm{kg} / \mathrm{m}^{2}\right)$ & $21.9(19.7-23.6)^{\mathrm{c}}$ & $22.4(20.1-24.4)^{\mathrm{c}}$ & 0.041 \\
\hline Subcutaneous fat: thick/normal or thin & $51 / 237$ & $84 / 313$ & 0.263 \\
\hline Wound protection: surgical drape/gauze/none & $282 / 3 / 3$ & $378 / 7 / 12$ & 0.156 \\
\hline Other organ resection: yes/no & $60 / 228$ & $15 / 382$ & $<0.0001$ \\
\hline Skin closure: subcuticular suture/staples & $145 / 143$ & $185 / 212$ & 0.333 \\
\hline Operation time $(\mathrm{min})$ & $260(220-296)^{\mathrm{c}}$ & $220(187-254)^{\mathrm{c}}$ & $<0.0001$ \\
\hline Estimated blood loss (g) & $341(200-603)^{\mathrm{c}}$ & $251(150-420)^{\mathrm{c}}$ & $<0.0001$ \\
\hline Duration of antibiotic prophylaxis: $\geqq 2$ days/1 day & $94 / 194$ & $103 / 294$ & 0.056 \\
\hline Drain: yes/no & $285 / 3$ & $313 / 84$ & $<0.0001$ \\
\hline Anticoagulation therapy: yes/no & $40 / 248$ & $50 / 347$ & 0.621 \\
\hline
\end{tabular}

Values in italics are statistically significant.

ASA American Society of Anesthesiology, BMI body mass index, CRP C-reactive protein, PNI prognostic nutritional index

a Data missing for one patient

b Data missing for 40 patients

${ }^{c}$ Median, with the interquartile range in parentheses 
Table 2 Univariate analysis of possible predictors of risk of superficial incisional surgical site infection (SSI) following gastrectomy

\begin{tabular}{|c|c|c|c|c|c|}
\hline \multirow[t]{2}{*}{ Variable } & \multirow{2}{*}{$\begin{array}{l}\text { Superficial insicional } \\
\text { SSI present }(n=42)\end{array}$} & \multirow{2}{*}{$\begin{array}{l}\text { Superficial insicional SSI } \\
\text { absent }(n=643)\end{array}$} & \multicolumn{3}{|c|}{ Univariate analysis } \\
\hline & & & $\mathrm{RR}$ & $95 \% \mathrm{CI}$ & $P$ \\
\hline Sex: male/female & $29 / 13$ & $455 / 188$ & 0.9 & $0.5-1.9$ & 0.814 \\
\hline Age (years): $\geqq 75 /<75$ & $15 / 27$ & $141 / 502$ & 2.0 & $1.0-3.8$ & 0.049 \\
\hline Hemoglobin (g/dl): $\geqq 10 /<10$ & $36 / 6$ & $569 / 74$ & 0.8 & $0.3-2.1$ & 0.598 \\
\hline $\mathrm{CRP}^{\mathrm{a}}(\mathrm{mg} / \mathrm{dl}): \geqq 0.1 /<0.1$ & $22 / 20$ & $365 / 277$ & 0.8 & $0.4-1.6$ & 0.572 \\
\hline PNI & $40 / 2$ & $603 / 40$ & 1.3 & $0.4-8.3$ & 0.692 \\
\hline $\operatorname{BMI}^{\mathrm{b}}\left(\mathrm{kg} / \mathrm{m}^{2}\right): \geqq 25 /<25$ & $7 / 33$ & $113 / 492$ & 0.9 & $0.4-2.0$ & 0.848 \\
\hline Subcutaneous fat: thick/normal or thin & $9 / 33$ & $126 / 517$ & 1.1 & $0.5-2.3$ & 0.775 \\
\hline Extent of gastric resection: total/distal & $15 / 27$ & $273 / 370$ & 0.8 & $0.4-1.4$ & 0.387 \\
\hline Other organ resection; yes/no & $5 / 37$ & $70 / 573$ & 1.1 & $0.4-2.7$ & 0.840 \\
\hline Skin closure: subcuticular suture/staples & $22 / 20$ & $308 / 335$ & 1.2 & $0.6-2.3$ & 0.574 \\
\hline Operation time $(\min ): \geqq 240 /<240$ & $21 / 21$ & $304 / 339$ & 1.1 & $0.6-2.1$ & 0.732 \\
\hline Estimated blood loss $(\mathrm{g}): \geqq 300 /<300$ & $21 / 21$ & $314 / 329$ & 1.0 & $0.6-2.0$ & 0.884 \\
\hline Duration of antibiotic prophylaxis (days): $\geqq 2 / 1$ & $14 / 28$ & $183 / 460$ & 1.3 & $0.6-2.4$ & 0.505 \\
\hline Drain: yes/no & $38 / 4$ & $560 / 83$ & 1.4 & $0.5-4.8$ & 0.508 \\
\hline Anticoagulation therapy: yes/no & $4 / 38$ & $86 / 557$ & 0.7 & $0.2-1.8$ & 0.456 \\
\hline Institution: high volume/low volume ${ }^{c}$ & $22 / 20$ & $388 / 255$ & 0.7 & $0.4-1.4$ & 0.315 \\
\hline
\end{tabular}

The value in italics is statistically significant.

$B M I$ body mass index, $C I$ confidence interval, $C R P$ C-reactive protein, $P N I$ prognostic nutritional index, $R R$ relative risk

${ }^{\text {a }}$ Data missing for one patient

b Data missing for 40 patients

${ }^{\mathrm{c}}$ High-volume institutions recruited more than 50 patients and low-volume institutions recruited fewer than 50 patients.

Table 3 Logistic regression model of prediction of risk of superficial incisional surgical site infection ( $S S I)$ following gastrectomy by age group

\begin{tabular}{llllll}
\hline Age (years) & $n$ & Superficial incisional SSI present & RR & $95 \%$ CI & $P$ \\
\hline$\leq 64$ & 248 & $11(4.4 \%)$ & \multicolumn{2}{l}{ Reference } & \\
$65-74$ & 281 & $16(5.7 \%)$ & 1.3 & $0.6-2.9$ & 0.510 \\
$75-84$ & 134 & $12(9.0 \%)$ & 2.1 & $0.9-5.1$ & 0.080 \\
$\geq 85$ & 22 & $3(13.6 \%)$ & 3.4 & $0.7-12.1$ & 0.110 \\
\hline
\end{tabular}

$C I$ confidence interval, $R R$ relative risk reducing the incidence of SSI. According to the clinical guideline for SSI of the National Collaborating Centre for Women's and Children's Health [9], age, underlying illness, obesity, smoking, and wound complications are significant risk factors for SSI.

We found that the incidence of superficial incisional SSI did not differ between total gastrectomy and distal gastrectomy. The National Healthcare Safety Network of the CDC in the USA has reported the overall incidence of SSI following gastric surgery, regardless of the type of gastric resection [2]. In contrast, the Japanese Healthcare Associated Infections Surveillance [3] has classified gastric surgery into three categories: namely, total, distal, and other gastrectomy. In the current study, we found that BMI, other organ resection, operation time, blood loss, and the presence of a drain differed significantly between total gastrectomy and distal gastrectomy. It therefore seems reasonable to assess total gastrectomy and distal gastrectomy as different procedures when evaluating surgical complications including SSIs, especially organ/space SSI. In this study, the incidence of organ/space SSI was $10.4 \%$ after total gastrectomy and $5.8 \%$ after distal gastrectomy (data not shown in "Results" because organ/space SSI was not clearly defined in the original study and there was considerable evaluation bias between participating institutions). To achieve an accurate evaluation, it is necessary to report the types of SSIs according to the type of surgery and to minimize bias. In contrast to superficial incisional SSI, there is a potential bias for evaluation of organ/space SSIs; thus, evaluation of severity using Dindo-Clavien scores is necessary.

The only significant risk factor for superficial incisional SSI that we identified was age of 75 years or older. One prospective observational study that assessed operative risk 
factors for SSI in patients undergoing total hip replacement reported that age older than 75 years was a significant risk factor [10]. One large prospective study $(n=23,649$ wounds) showed a broad trend toward increasing incidence of SSI with increasing age when the patients were stratified by age group [11]. We also found that the incidence of superficial incisional SSI tended to increase in parallel with age. To reduce the risk of superficial incisional SSI, several publications have proposed procedures involving skin preparation, barrier retractional wound protection, and pulsatile irrigation of wounds before closure [12]. These procedures are now routinely applied during gastrointestinal surgery. Thus, in this study, procedures for wound protection were well controlled, $3 \mathrm{M}^{\mathrm{TM}}$ Steri-Drape ${ }^{\mathrm{TM}}$ wound edge protector (3M, St Paul, MN, USA) having been used in 13 institutions and Alexis ${ }^{\circledR}$ wound protector/ retractor (Applied Medical, Rancho Santa Margarita, CA, USA) having been in a further ten of the 24 participating institutions. No wound protection was used in the remaining institution. We found no difference between these two types of wound protection (data not shown). Therefore, we conclude that advanced age is a risk factor for superficial incisional SSI after gastrectomy regardless of the skin closure method and the type of wound protector.

Being overweight is reportedly a risk factor for SSI and other surgical complications [13, 14]. Very low BMI and very high BMI are well-acknowledged risk factors [15, 16]. When we analyzed the incidence of superficial incisional SSIs in four BMI categories, we found no significant differences between the groups, indicating that BMI may not be a risk factor for superficial incisional SSI after gastrectomy.

In conclusion, the incidence of superficial incisional SSI does not differ between total gastrectomy and distal gastrectomy. Advanced age is a risk factor for superficial incisional SSI after gastrectomy.

Acknowledgments This study was performed by the Clinical Study Group of Osaka University on Section of Risk Management (CSGORM) and included the following institutions: Hannan Chuo Hospital, Higashiosaka City General Hospital, Ikeda City Hospital, Itami City Hospital, Kansai Rosai Hospital, Japan Community Health Care Organization Osaka Hospital (former name Osaka-Kouseinenkin Hospital), Japan Community Health Care Organization Osaka Minato Central Hospital (former name Osaka Seamen's Insurance Hospital), Kinki Central Hospital of the Mutual Aid Association of Public School Teachers, Moriguchi Keijinkai Hospital, National Hospital Organization Kure Medical Center, National Hospital Organization Osaka National Hospital, Nishinomiya Municipal Central Hospital, Nissay Hospital, NTT WEST Osaka Hospital, Osaka General Medical Center, Osaka Medical Center for Cancer and Cardiovascular Diseases, Osaka Rosai Hospital, Osaka University, Otemae Hospital, Rinku General Medical Center, Sakai Municipal Hospital, Tane General Hospital, Toyonaka Municipal Hospital, and Yao Municipal Hospital.

Conflict of interest The authors declare no conflicts of interest.

\section{References}

1. Mangram AJ, Horan TC, Pearson ML, Silver LC, Jarvis WR. Guideline for prevention of surgical site infection. Infect Control Hosp Epidemiol. 1999;20:250-78.

2. Mu Y, Edwards JR, Horan TC, Berrios-Torres SI, Fridkin SK. Improving risk-adjusted measures of surgical site infection for the national healthcare safety network. Infect Control Hosp Epidemiol. 2011;32:970-86.

3. Japanese Healthcare Associated Infections Surveillance. Annual SSI surveillance report. http://www.kankyokansen.org/common/ fckeditor/editor/filemanager/connectors/php/transfer.php?file=/ JSEI/uid000003_6A686169735F7373692D73756D6D61727928 32292E706466. Accessed Jan 2015.

4. Fujitani K, Tsujinaka T, Fujita J, Miyashiro I, Imamura H, Kimura Y, et al. Prospective randomized trial of preoperative enteral immunonutrition followed by elective total gastrectomy for gastric cancer. Br J Surg. 2012;99:621-9.

5. Imamura H, Kurokawa Y, Tsujinaka T, Inoue K, Kimura Y, Iijima $\mathrm{S}$, et al. Intraoperative versus extended antimicrobial prophylaxis after gastric cancer surgery: a phase 3, open-label, randomised controlled, non-inferiority trial. Lancet Infect Dis. 2012;12:381-7.

6. Tsujinaka T, Yamamoto K, Fujita J, Endo S, Kawada J, Nakahira $\mathrm{S}$, et al. Subcuticular sutures versus staples for skin closure after open gastrointestinal surgery: a phase 3, multicentre, open-label, randomised controlled trial. Lancet. 2013;382:1105-12.

7. Onodera T, Goseki N, Kosaki G. Prognostic nutritional index in gastrointestinal surgery of malnourished cancer patients (in Japanese with English abstract). J Jpn Surg Soc. 1984;85:1001-5.

8. Endo S, Yoshikawa Y, Hatanaka N, Tominaga H, Shimizu Y, Hiraoka K, et al. Treatment for gastric carcinoma in the oldest old patients. Gastric Cancer. 2011;14:139-43.

9. National Collaborating Centre for Women's and Children's Health. Surgical site infection: prevention and treatment of surgical site infection. London: RCOG Press; 2008. p. 15-20.

10. Ridgeway S, Wilson J, Charlet A, Kafatos G, Pearson A, Coello R. Infection of the surgical site after arthroplasty of the hip. J Bone Joint Surg Br. 2005;87:844-50.

11. Cruse PJ. Foord R. A five-year prospective study of 23,649 surgical wounds. Arch Surg. 1973;107:206-10.

12. Detsky AS, McLaughlin JR, Baker JP, Johnston N, Whittaker S, Mendelson RA, et al. What is subjective global assessment of nutritional status? J Parenter Enteral Nutr. 1987;11:8-13.

13. Tsujinaka T, Sasako M, Yamamoto S, Sano T, Kurokawa Y, Nashimoto A, et al. Influence of overweight on surgical complications for gastric cancer: results from a randomized control trial comparing D2 and extended para-aortic D3 lymphadenectomy (JCOG9501). Ann Surg Oncol. 2007;14:355-61.

14. Hirao M, Tsujinaka $\mathrm{T}$, Imamura $\mathrm{H}$, Kurokawa $\mathrm{Y}$, Inoue $\mathrm{K}$, Kimura $\mathrm{Y}$, et al. Overweight is a risk factor for surgical site infection following distal gastrectomy for gastric cancer. Gastric Cancer. 2013;16:239-44.

15. Cho M, Kang J, Kim IK, Lee KY, Sohn SK. Underweight body mass index as a predictive factor for surgical site infections after laparoscopic appendectomy. Yonsei Med J. 2014;55:1611-6.

16. Esemuede IO, Murray AC, Lee-Kong SA, Feingold DL, Kiran RP. Obesity, regardless of comorbidity, influences outcomes after colorectal surgery-time to rethink the pay-for-performance metrics? J Gastrointest Surg. 2014;18:2163-8. 